\title{
Keramiken und Kunststoffe verdrängen zunehmend Metalle
}

\begin{abstract}
„Metalle im Mund: Brauchen wir sie noch? Können sie krank machen?" Diese Fragen hat die Deutsche Gesellschaft für Zahn-, Mund- und Kieferheilkunde (DGZMK) Mitte Mai auf einer wissenschaftlichen Pressekonferenz in Berlin aus verschiedenen Blickwinkeln beleuchtet. Wie DGZMK-Präsident, Prof. Dr. Michael Walter, einleitend betonte, seien im Gegensatz zur weit verbreiteten Wahrnehmung die Risiken, die von Metallen im Mund ausgehen, aus wissenschaftlicher Perspektive eher gering. Daher sprach er sich für einen "faktenbasierten Umgang" mit diesem Thema aus. „Keramiken und Kunststoffe können Metalle heute bei vielen Therapien schon ersetzen, ganz verdrängen können sie diese aber noch nicht", sagte Walter.

Mit Blick auf die Verträglichkeit von Metallen klärte Priv.-Doz. Dr. Anne Wolowski vom Arbeitskreis Psychologie und Psychosomatik in der DGZMK auf, dass die Wahrnehmung eines Allergierisikos auf Metalle in der Bevölkerung im Widerspruch zu wissenschaftlichen Forschungsergebnissen stehe. Unverträglichkeitsreaktionen seien eher selten. Die Zahnmedizinerin vom Universitätsklinikum Münster rät bei unklaren Beschwerden wie Müdigkeit und Mundtrockenheit oder dem Verdacht auf eine Unverträglichkeit unbedingt zur Zusammenarbeit zwischen Zahnärzten und Allgemeinmedizinern. Diagnostik und weitere Therapie seien unter anderem abhängig von der Art der Beschwerden, der Vorgeschichte und vorliegender körperlicher Befunde. Zudem sollte eine vorschnelle und von Polemik gesteuerte Diagnostik vermieden werden. Unsicherheiten durch unbegründete Spekulationen würden zur Überschätzung eines objektiven Risikos führen. Dieser sogenannte Noceboeffekt belaste Betroffene unnötig in hohem Maße, so Wolowski.
\end{abstract}

\section{Wunsch nach mehr Ästhetik verdrängt Amalgam}

Prof. Dr. Roland Frankenberger von der Deutschen Gesellschaft für Zahnerhaltung widmete sich in seinem Vortrag dem Fül-

\section{Landarztmangel \\ Bayern zeigt sich großzügig}

Dass es immer weniger junge Ärzte und Zahnärzte aufs Land zieht, ist eine Tatsache, der viele Politiker bereits ins Auge sehen. Bayerns Gesundheitsministerin Melanie Huml (CSU) will künftig mit mehr Geld mehr junge Ärzte in die ländlichen Gebiete locken. Statt bisher 300 Euro sollen Stipendiaten, die sich verpflichten, nach dem Studium als Arzt im ländlichen Raum tätig zu werden, in Zukunft 500 Euro monatlich bekommen, sagte die Ministerin laut Ärztenachrichtendienst bei der Landesversammlung des Hartmannbundes in Bamberg. Der Freistaat fördere zudem die Niederlassung von Ärzten in Kleinstädten mit bis zu 60.000 Euro.

Allein an den Finanzen hängt das Problem der ärztlichen und zahnärztlichen Landflucht nach Auffassung des Freien Verbandes Deutscher Zahnärzte (FVDZ) allerdings nicht. „Wir müssen vielmehr die Rahmenbedingungen verbessern, die eine Niederlassung auf dem Land wieder attraktiv machen und heutigen Lebensentwürfen junger Ärzte und Zahnärzte lungsmaterial Amalgam, das wegen seines 50-prozentigen Quecksilberanteils immer wieder in der Kritik steht. Er erklärte, dass der Trend aus ästhetischen Gründen zunehmend weg vom Amalgam hin zu minimalinvasiven Füllungstechniken gehe. „Daher haben die Komposite das Amalgam heute de facto als Massenfüllungsmaterial abgelöst", sagte Frankenberger. Aus toxikologischen Gründen auf Amalgam zu verzichten und auf Ersatzmaterialien zurückzugreifen, sieht Frankenberger nicht als notwendig an.

„Ein perfekt biokompatibles Füllungsmaterial gibt es nicht. Es gilt daher, eine stringente Risikoabschätzung durchzuführen. Nach dieser ist die Verwendung von Amalgam und Amalgamersatzmaterialien in der Regel unbedenklich. Das heißt, das Risiko ist akzeptabel.“

\section{Titanimplantate sind nach wie vor Goldstandard}

Prof. Dr. Stefan Wolfart von der Deutschen Gesellschaft für Prothetische Zahnmedizin und Biomaterialien betonte, dass der Einsatz von Metallen für Zahnersatzversorgungen stark rückläufig sei. Moderne Keramiken könnten jedoch nicht überall als Ersatz dienen. „Ganz ohne Metalle geht es vor allem bei großen Brücken, in der Implantatprothetik und bei herausnehmbaren Prothesen noch nicht", sagte Wolfart.

Bei Zahnlosigkeit dienen zahnärztliche Implantate, die überwiegend aus Titan gefertigt werden, als Therapiealternative. „Die weitgehend problemlose Verwendung von Titanimplantaten ist wissenschaftlich bestens belegt", bestätigte Prof. Dr. Frank Schwarz, Präsident der Deutschen Gesellschaft für Implantologie. Aktuell seien Titanimplantate der „Goldstandard“. Keramikimplantate können aus Sicht von Schwarz bei bestimmten Indikationen eingesetzt werden. Aber hier müsse die Forschung noch weitergehen, und die Entwicklung bleibe abzuwarten.

Carolin Stehr

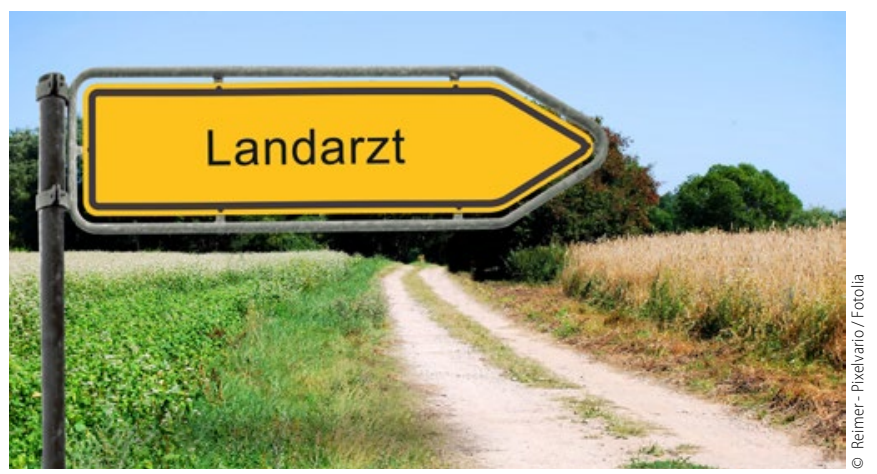

entspricht", sagte der FVDZ-Bundesvorsitzende Harald Schrader. Der Freie Verband entwickelt derzeit das Modell genossenschaftlich betriebener Praxen, die gerade für die junge Zahnärzte- und Ärztegeneration gute Möglichkeiten bieten, ohne hohen finanziellen Einsatz freiberuflich in der Praxis tätig zu sein. „Die Angebote aus der Politik können dabei allerdings Hand in Hand gehen, mit Angeboten, die aus dem Berufsstand kommen“, sagte Schrader. 